\title{
Sonographic images of hepato-pancreatico-biliary and intestinal ascariasis: A pictorial review
}

\author{
Donboklang Lynser ${ }^{1} \cdot$ Akash Handique $^{2}$ - Chhunthang Daniala ${ }^{2}$ - Pranjal Phukan ${ }^{2}$. \\ Evarisalin Marbaniang ${ }^{3}$
}

Received: 29 April 2015 /Revised: 5 August 2015 / Accepted: 3 September 2015 /Published online: 16 September 2015

(C) The Author(s) 2015. This article is published with open access at Springerlink.com

\begin{abstract}
Despite advancement in the diagnosis and treatment of intestinal helminthiasis, ascariasis remains the most common cause of helminthic infections in the developing countries. Ultrasound offers a rapid, safe, and noninvasive approach to the diagnosis of intestinal ascariasis. Ultrasound is also the modality of choice for diagnosis of hepatobiliary ascariasis, which is relatively rare and is due to migration of intestinal worms through the papilla of Vater. We present an imaging spectrum of hepato-pancreatico-biliary and intestinal ascariasis.

Main messages

- Ascariasis refer to infestation by the round worm ascaris lumbricoides.

- Ascaris eggs are excreted in faeces and are infective to humans.

- Eggs hatch to larva, travel to the lungs and mature to adult worms.

- Intestinal obstruction can be caused by multiple ascariasis forming bag of worms.
\end{abstract}

Donboklang Lynser

bokdlynser@yahoo.co.in

1 Department of Radiology and Imaging, Ganesh Das Hospital, Lawmali, Shillong 793001, Meghalaya, India

2 Department of Radiology and Imaging, North Eastern Indira Gandhi Regional Institute of Health and Medical Sciences, Mawdiangdiang, Shillong 793018, Meghalaya, India

3 Department of Pathology, North Eastern Indira Gandhi Regional Institute of Health and Medical Sciences, Mawdiangdiang, Shillong 793018, Meghalaya, India
Keywords Ascariasis · Ultrasound · Acute abdomen · Cholecystitis $\cdot$ Pancreatitis

Ascariasis is a common problem, with approximately one-fourth of the world population infected [1]. The majority of infections occur in the developing countries of Asia and Latin America [2]. Ascariasis refers to infestation by the roundworm Ascaris lumbricoides. Humans become infested after ingesting material contaminated with embryonated eggs from faeces of an infected individual. The eggs hatch to larvae in the small intestine under the influence of gastric secretions. The larvae then penetrate the intestinal mucosa and are transported haematogenously to the lungs. The worm then matures in the alveoli and then travels up the bronchi and trachea only to be swallowed again. Once in the intestine, they mature to adult worms. They then mate and produce eggs. The eggs are then excreted along with faeces and are infective to humans, thereby completing the cycle.

\section{Ultrasound imaging of intestinal ascariasis}

Adult worms are seen as tubular structures outlined by intestinal fluid. The adult worm is seen as a large, curved echogenic strip with an inner, anechoic, longitudinal canal [3]. When we used a high-resolution linear (7-10 mhz) transducer (Logiq P5, GE Milwaukee US), in long section the worm appeared as four parallel lines separated by three anechoic bands (Fig. 1a). In cross section, it is round and sometimes appearing as a "target sign" [4]. The live worms are sometimes seen moving in the intestinal lumen. At times they are 

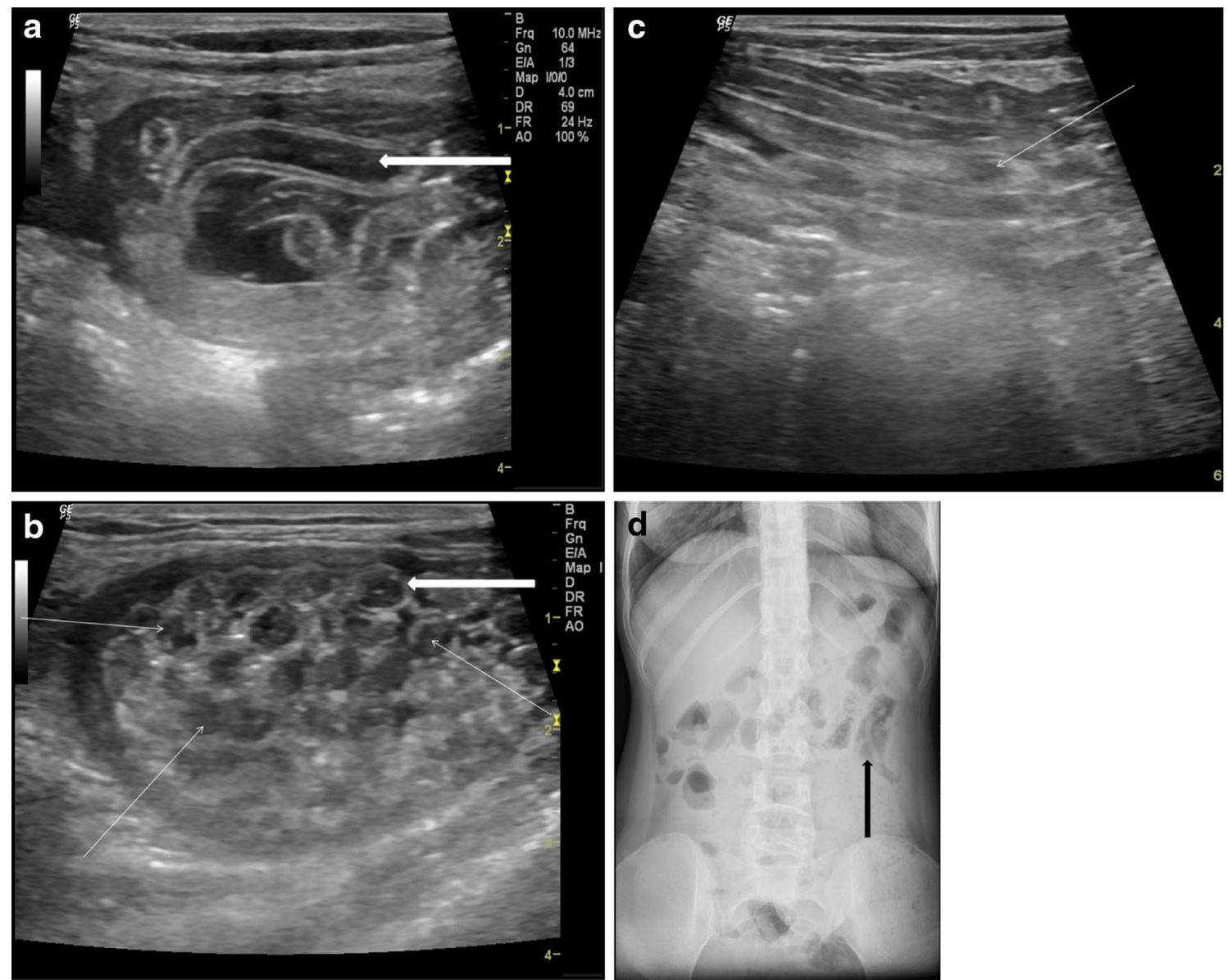

Fig. 1 A 25-year-old female with acute abdomen and clinical signs of intestinal obstruction. (a) High resolution ultrasound using linear array transducer showing intestinal ascariasis, note distended fluid filled alimentary canal of the ascaris (thick white arrow), the "inner tube sign", (b) same patient showing multiple ring like shadows (thin

white arrows), some with target appearance (thick white arrow) on transverse section of the intestinal lumen indicating bag of worms, (c) "Bag of worms" in a long section (thin white arrow) and (d) PA erect X-ray abdomen of this patient showing tubular soft densities suggestive of ascariasis (thick black arrow)

Fig. 2 A patient with acute epigastrium, CBD showing double linear echogenic wall indicating ascariasis"strip sign" (white arrow)

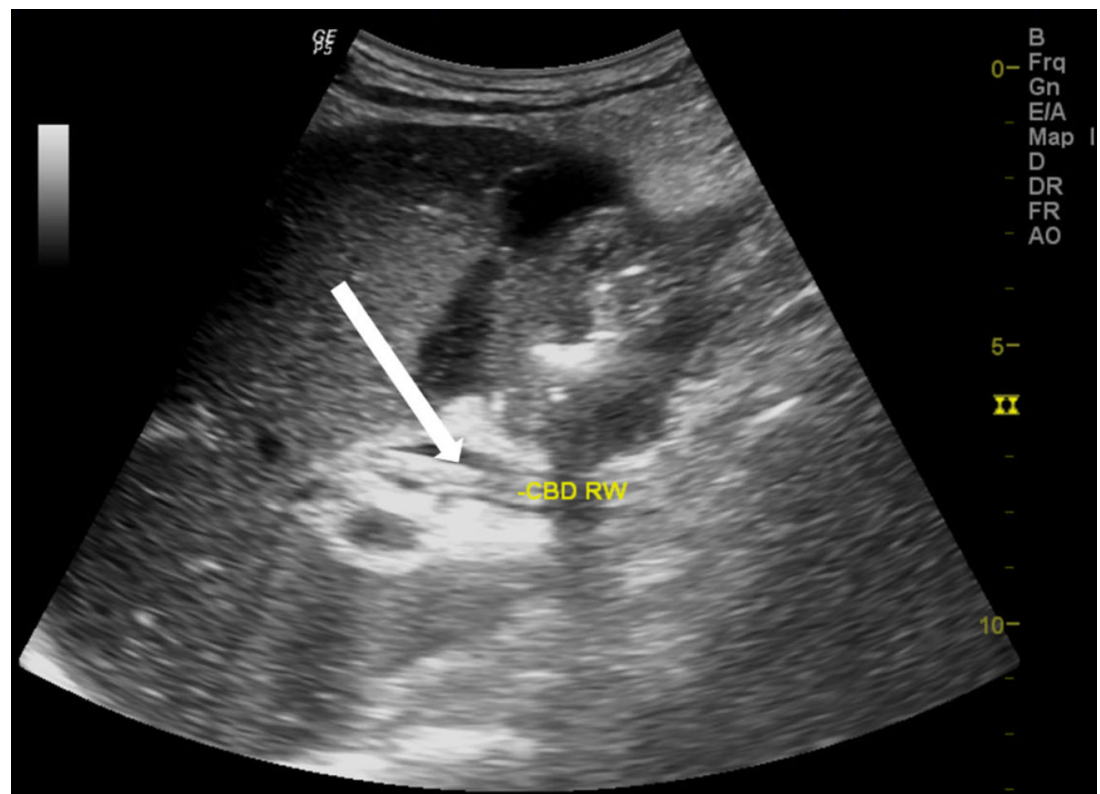


multiple forming a bag or cluster of worms, which can cause intestinal obstruction (Fig. $1 \mathrm{~b} \& \mathrm{c}$ ). On rare occasions, the round worm can also be seen in a plain x-ray abdomen (Fig. 1d).

\section{Hepatobiliary ascariasis}

Although no reliable data exists, hepatobiliary ascariasis is far less common compared to intestinal ascariasis alone. Patients with hepato-biliary ascariasis can present as biliary colic, acute cholecystitis, acute cholangitis, acute pancreatitis, and hepatic abscess. They may also present later as intrahepatic duct calculi due to recurrent biliary invasion [5]. Ascariasis in the
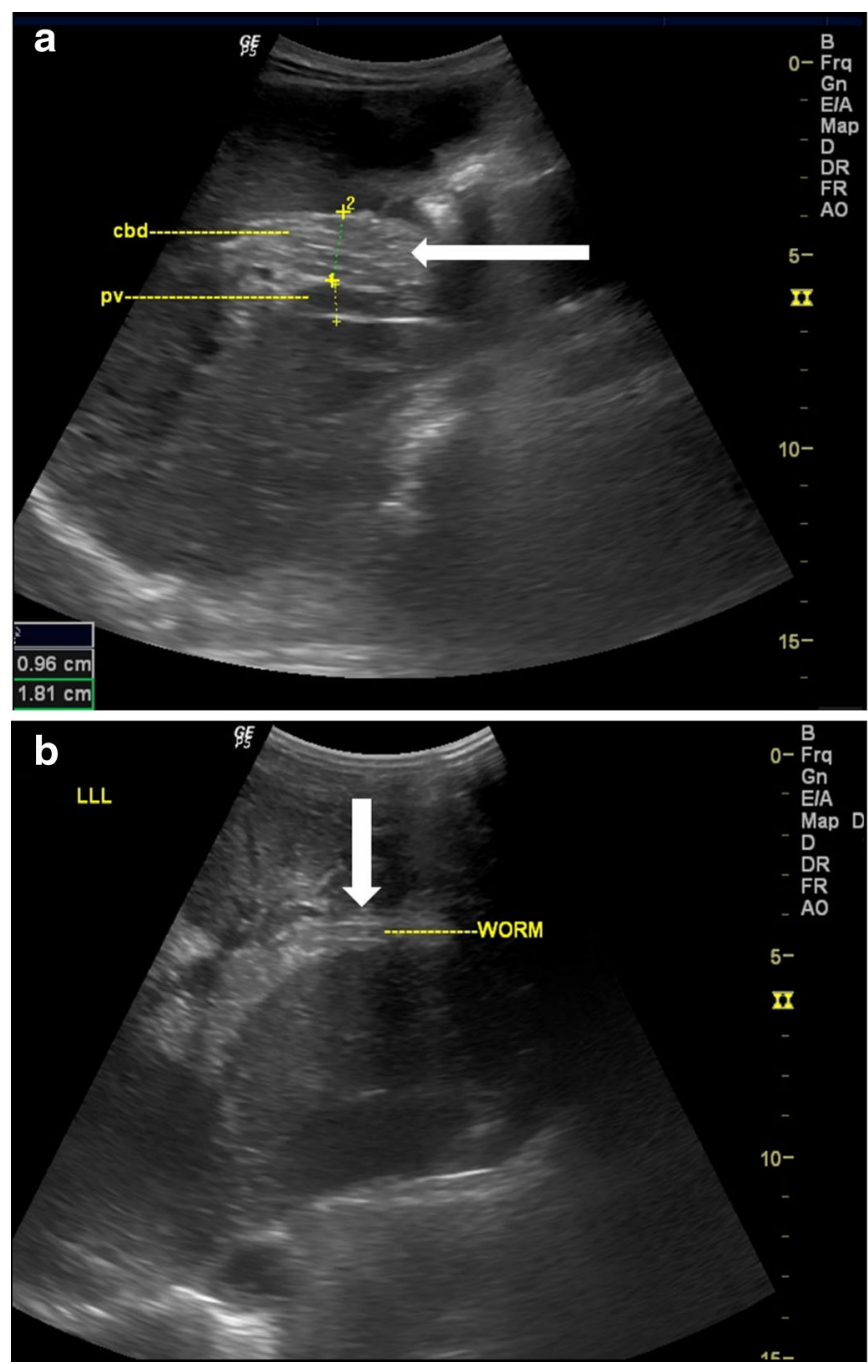

Fig. 3 A 14-year-old female with acute biliary colic. (a) Multiple ascaris in CBD, the spaghetti sign, CBD is filled with multiple worms (long thick white arrow), (b) intra hepatic duct of the left lobe of liver showing a double linear echogenic wall indicating ascariasis (white arrow) and (c) biliary tract can have any of the following imaging features on sonography [6].

1. Inner-tube sign: The roundworm may be seen as a thick echoic stripe with a central, longitudinal anechoic tube (gastrointestinal tract of the worm) in a gall bladder or CBD.

2. Coil of worm in the gall bladder.

3. Strip sign: Thin non-shadowing strip without an inner tube in the CBD or gall bladder.

4. Spaghetti sign: Overlapping longitudinal interfaces in the main bile duct.

5. In our observation, we have found several linear calcified structures within the bile ducts that might be calcified dead worms, possibly representing remote hepatobiliary infestation.

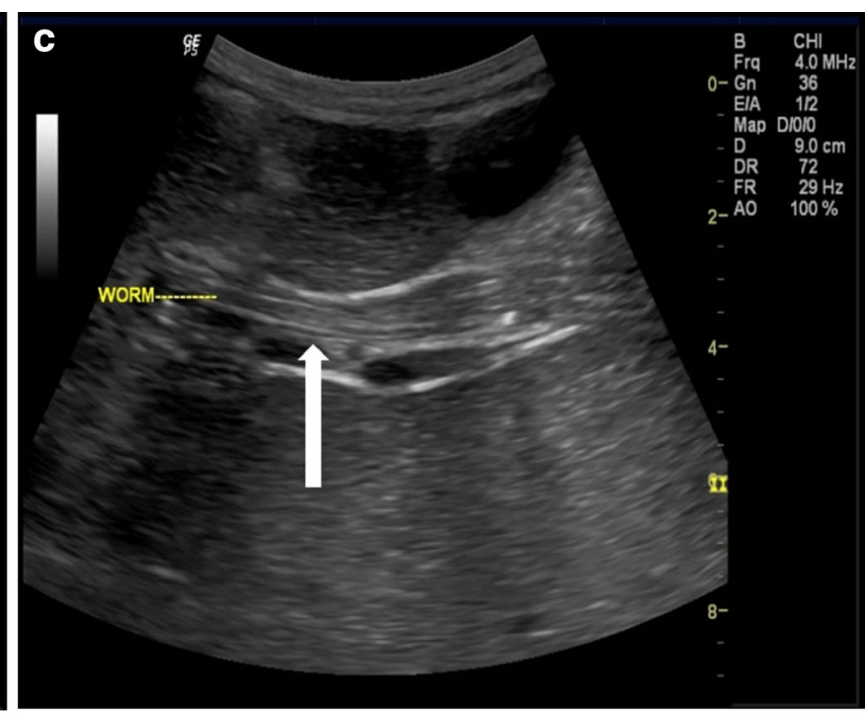

following antihelminthic medication, sonography was repeated the next day showing reduction in the number of worms. Note double echogenic walls (strip sign) of CBD round worm (white arrow) 
Fig. 4 A 35-year-old male with acute abdomen showing pseudotumour-like shadows in the CBD (thick white arrow). Note that the spaghetti sign is seen in the long section (thin white arrow)

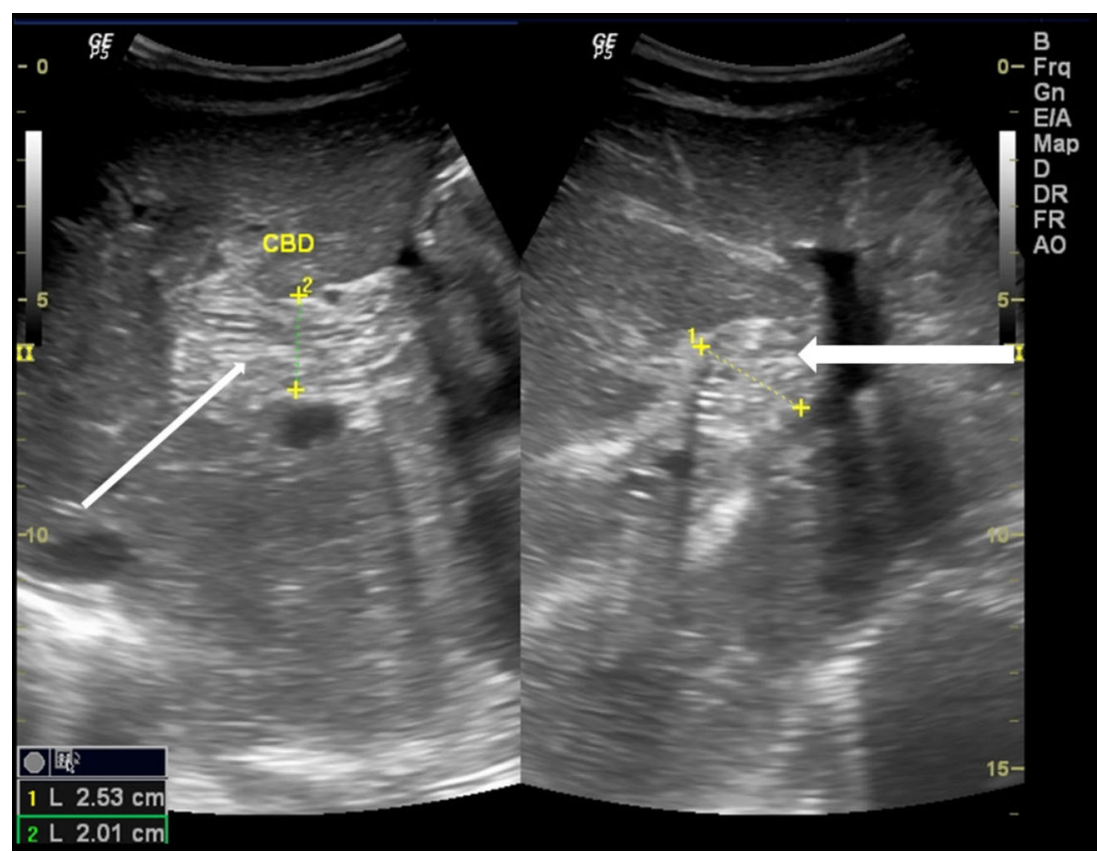

\section{Common bile duct (CBD)}

The worm in the CBD can be seen as single or multiple tubular, linear echogenic non-shadowing walls (strip sign) (Fig. 2).
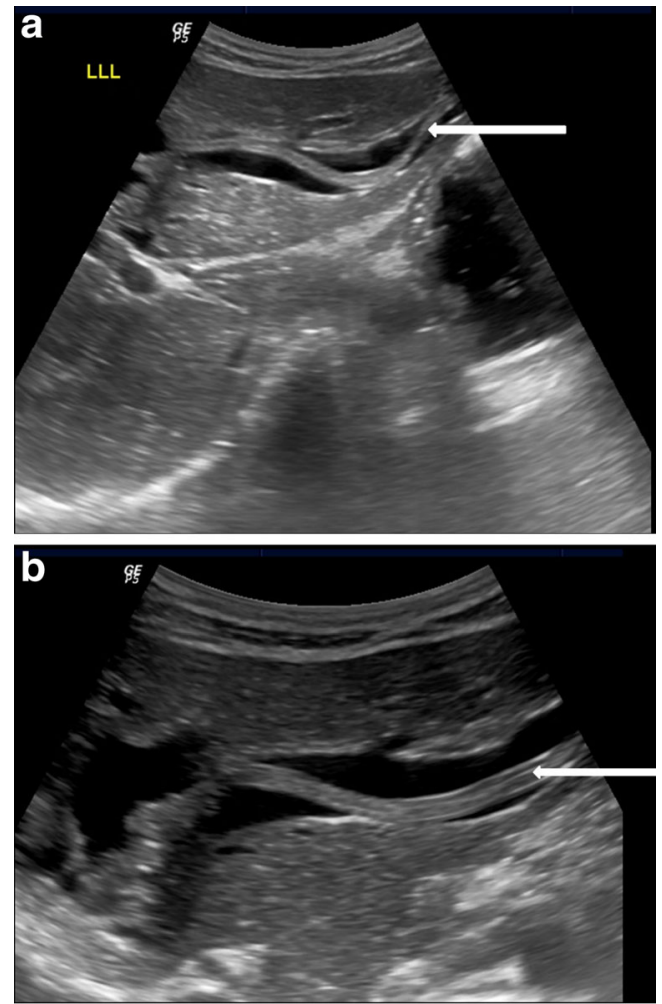

Fig. 5 A 35-year-old female with acute pain in the right hypochondrium. (a) Ascaris seen in the left dilated intrahepatic duct (white arrow) and (b) magnified view of the ascaris in the dilated left intrahepatic duct, triple line seen (white thick arrow)
On rare occasions, too many worms inside the CBD can appear as multiple echogenic non-shadowing linear interfaces, giving a classical spaghetti sign (Fig. $3 \mathrm{a}, \mathrm{b}$ and c). CBD round worms sometimes has a pseudotumour appearance (Fig. 4). $\mathrm{CBD}$ and intrahepatic ducts can become very dilated; however, obstructive jaundice is exceedingly rare.

\section{Intrahepatic ducts}

Ascaris in the intrahepatic ducts are less common than the common bile duct. They may be seen in either lobes of liver

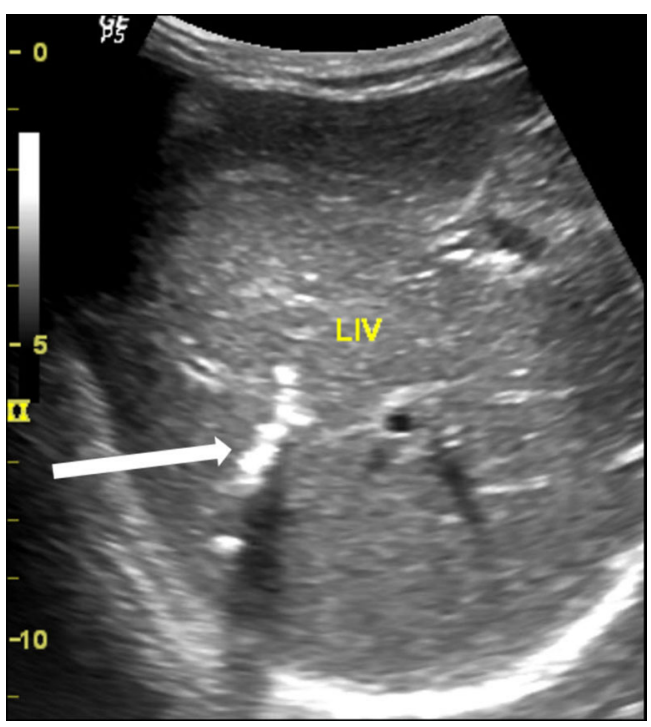

Fig. 6 A 40-year-old female showing calcified shadow indicating calcified worms in the intrahepatic duct of the right lobe of liver (thick white arrow) 
Fig. 7 A 25-year-old female showing coil of ascariasis inside the gall bladder lumen (white arrow)

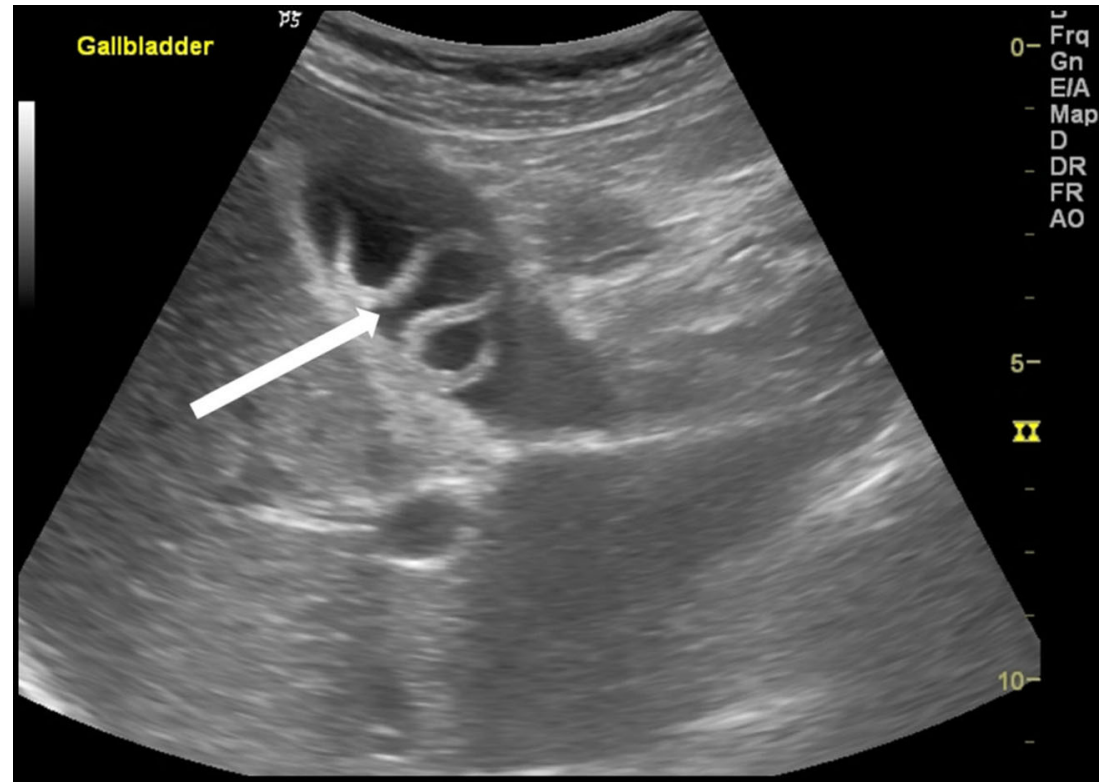

with intrahepatic biliary dilatation. They are seen as similar tubular structures inside the hepatic ducts with a "strip sign" (Fig. 5a). On rare occasion, a "triple line" can also be appreciated on magnified view or with the use of high resolution linear array transducer (Fig. 5b). Calcified worms in the intrahepatic duct are also occasionally seen in asymptomatic patients on routine sonography (Fig. 6).

\section{Gall bladder}

Ascaris in the gallbladder is rare, constituting $2.1 \%$ of hepatobiliary ascariasis [7]. This can be easily picked up on ultrasound. Inner tube may not be seen (Strip sign). Live

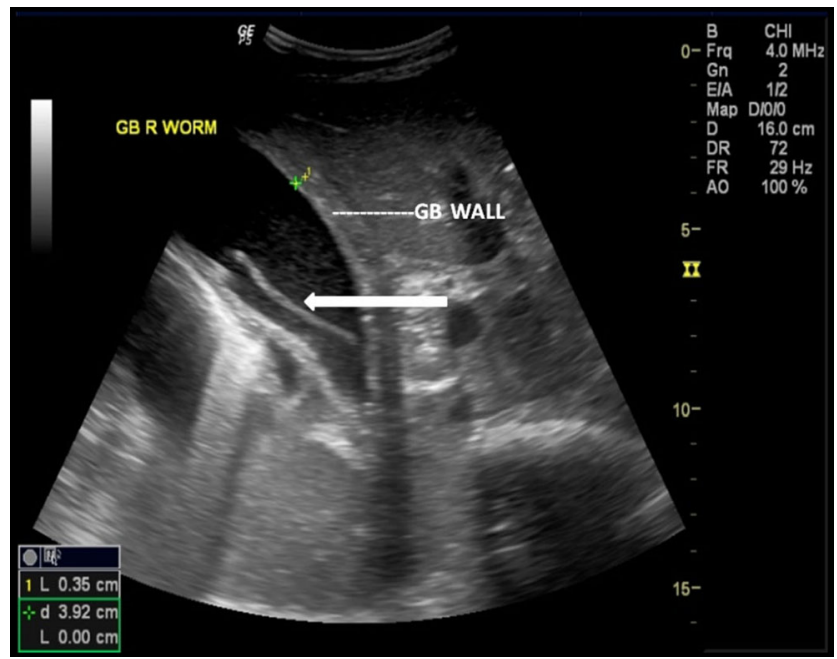

Fig. 8 A 25-year-old male with acute right hypochondriac pain showing ascaris (thick white arrow) inside the gall bladder. Note GB wall thickening and luminal sludge indicating acute cholecystitis worms may move within the gall bladder. The ascaris may be seen as a coil of worm in the gall bladder lumen (Fig. 7). The worm in the gall bladder may also present with cholecystitis (Fig. 8).

\section{Pancreatic duct}

Rarely, ascaris in the main pancreatic duct can present with pancreatitis. The main pancreatic duct may be dilated, with "triple line sign", "strip sign" or an "inner tube sign" and occasional pancreatic oedema (Fig. 9).

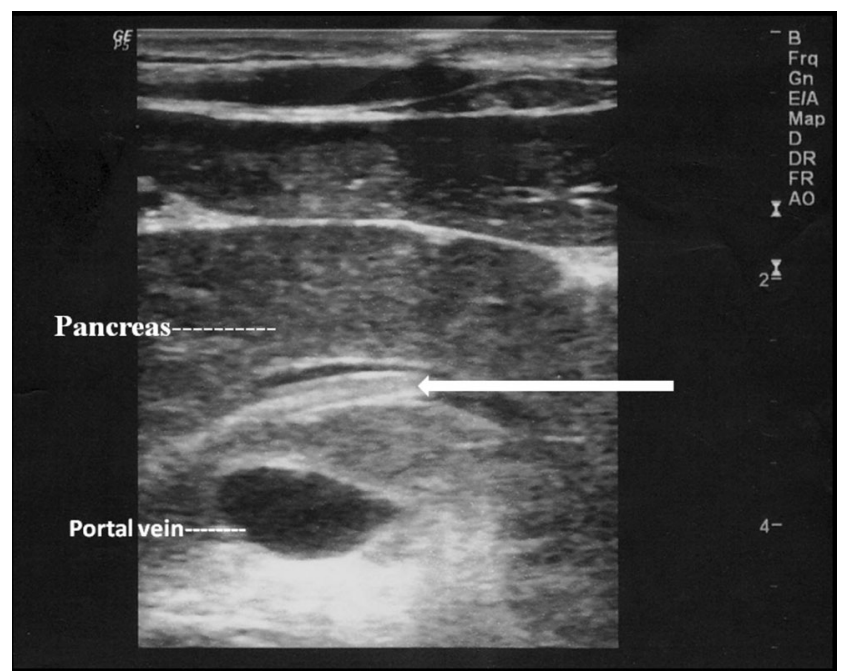

Fig. 9 A 15-year-old male presented with acute epigastric pain and elevated pancreatic enzymes. High resolution ultrasound using linear array transducer shows ascaris with triple line within in the dilated main pancreatic duct (long white arrow) with pancreatitis 


\section{Discussion}

Multiple Ascaris Lumbricoides round worms can result in intestinal obstruction, or even cause volvulus with consequent necrosis of the involved bowel segment [8]. Magnetic resonance cholangio-pancreatography (MRCP) also has a role in the diagnosis of hepatobiliary ascariasis [9], and though other parasites can be involved in the biliary system [10], ultrasound with all its typical imaging features has a definite role in the diagnosis of hepato-pancreatico-biliary and intestinal ascariasis.

\section{Conclusion}

In conclusion, clean hygiene prevents contamination of foods by ascaris eggs, therefore arresting the natural cycle. Sonography, on the other hand, is a noninvasive, cheap, rapid and safe imaging modality in hepato-pancreatico-biliary and intestinal ascariasis, especially in situations where other types of imaging are costly or inaccessible.

\footnotetext{
Aknowledgments To the staff of the Department of Radiology and Imaging, at Ganesh Das Hospital and NEIGRIHMS Hospital for their immense help
}

\section{Conflict of interest statement and financial assistance None.}

Open Access This article is distributed under the terms of the Creative Commons Attribution 4.0 International License (http://creativecommons.org/licenses/by/4.0/), which permits unrestricted use, distribution, and reproduction in any medium, provided you give appropriate credit to the original author(s) and the source, provide a link to the Creative Commons license, and indicate if changes were made.

\section{References}

1. Gabaldon A et al (1967) Control of ascariais (report of a WHO expert committee). World Health Organ Tech Rep Ser 379:6-7

2. Khuroo MS (1996) Ascariasis. Gastroenterol Clin North Am 25(3): 553-577

3. Hoffmann $\mathrm{H}$ et al (1997) In vivo and in vitro studies of the sonographic detection of Ascaris lumbricoides. Pediatr Radiol 27(3): 226-229

4. Mahmood T et al (2001) Ultrasonographic appearance of ascaris lumbricoides in the small bowel. J Ultrasound Med 20:269-274

5. Khuroo MS, Zargar SA, Mahajan R (1990) Hepatobiliary and pancreatic ascariasis in India. Lancet 335:1503-1506

6. Schulman A et al (1982) Sonographic diagnosis of biliary ascariasis. AJR 139:485-489

7. Khuroo MS et al (1992) Sonographic findings in gallbladder ascariasis. J Clin Ultrasound 20(9):587-591

8. Rodriguez EJ et al (2003) Ascariasis causing small bowel volvulus. Radiographics 23(5):1291-1293

9. Hashmi MA, De JK (2009) Biliary ascariasis on magnetic resonance cholangiopancreatography. J Global Infect Dis 1(2):144-145

10. Lim JH, Kim SY, Park CM (2007) Parasitic diseases of the biliary tract. AJR 188(6):1596-1603 